\title{
Diffraction Resistant Scalar Beams Generated by a Parabolic Reflector and a Source of Spherical Waves
}

\author{
Michel Zamboni-Rached* \\ Department of Electrical and Computer Engineering at the University of Toronto, \\ Toronto, ON, Canada. \\ Mariana Carolina de Assis \\ DECOM-FEEC, Universidade Estadual de Campinas, SP, Brazil. \\ and \\ Leonardo A. Ambrosio \\ Department of Electrical and Computer Engineering, São Carlos School of Engineering, \\ University of São Paulo, SP, Brazil
}

\begin{abstract}
In this work, we propose the generation of diffraction resistant beams by using a parabolic reflector and a source of spherical waves positioned at a point slightly displaced from its focus (away from the reflector). In our analysis, considering the reflector dimensions much greater than the wavelength, we describe the main characteristics of the resulting beams, showing their properties of resistance to the diffraction effects. Due to its simplicity, this method may be an interesting alternative for the generation of long range diffraction resistant waves.
\end{abstract}

\section{Introduction}

The phenomenon of diffraction causes gradual spatial broadening of beams and pulses during propagation, and it can be a limiting factor for any application where it is desired that the wave maintains its transverse localization, such as in optical tweezers, remote sensing, tissue

(†) Corresponding author: mzamboni@decom.fee.unicamp.br

* On leave from DECOM-FEEC, University of Campinas 
characterization, free space optics communications (FSO), optical atom guiding, among others $[1,9]$.

Ideal non-diffracting waves (also called localized waves) form a set of free space solutions of the linear wave equation, $\left(\nabla^{2}-\partial_{c t}{ }^{2}\right) \Psi=0$, whose main characteristic is the total resistance to the diffraction effects. But, for these waves, the power flow through any plane perpendicular to the propagation direction is infinite, thus making impossible their experimental generation. However, it is possible to construct, theoretically and experimentally, truncated versions of these ideal beams[2], presenting finite power flow and resistance to the diffraction effects for long (finite) distances.

In this paper we propose a simple way to obtain beams capable of maintaining their transverse spot sizes for distances considerably greater than those presented for the usual beams, like the gaussian ones. For this purpose, we use a parabolic reflector and a source of spherical waves positioned at a point slightly displaced from its focus (away from the reflector) - that, from now on, we will mention as the "paraboloid setup".

It is important to note that we perform a simplistic analysis, assuming that the field in question is a scalar obeying the usual wave equation. We also assume that the source, placed at a point slightly shifted from the focus, produces a scalar spherical (isotropic) wave and that the whole phenomenon, including the reflected field, possesses azimuthal symmetry. Such assumptions may be inaccurate in practice, as the vector field character of the case considered here is important and even if we consider this scalar field as one of the transverse Cartesian field components, it would hardly possess azimuthal symmetry. Even so, the reason for which we adopted a scalar analysis here is that, in addition to simplicity, our intention is just to provide a strong evidence of the possibility of generating diffraction resistant beams through the use of parabolic reflectors.

A more complete and rigorous analysis of the setup proposed here, taking into account the vectorial nature of the fields, will be addressed in a future paper.

In Section 2 we present some important concepts used through the paper, like the Gaussian beam solution, the zero order Bessel beam solution (one of the most important non-diffracting 
beams) and its truncated version, the Fresnel diffraction integral, etc..

Section 3 is devoted to the theoretical description of the beams generated by the paraboloid setup. We start with an analytical and approximate description of some general properties of the considered beams, which are then calculated through numerical simulation of the Fresnel diffraction integral, with examples in the optical and microwave frequency ranges. The resulting beams are resistant to the diffraction effects for considerable distances when compared with ordinary gaussian beams.

Sections 4 and 5 are devoted to the conclusions and acknowledgments, respectively.

We believe that, due to its simplicity, the method presented here can be a very interesting alternative for the generation of long range diffraction resistant beams in optics and microwave applications.

\section{Some important concepts}

Let us consider the free space and a field, $\Psi$, governed by the wave equation $\dagger$. In the paraxial approximation, a possible monochromatic solution to this wave field is the the Gaussian beam solution[1]:

$$
\Psi(\rho, z, t)=\frac{2 q^{2} \exp \left[\frac{-\rho^{2}}{4\left(q^{2}+i z / 2 k\right)}\right]}{2\left(q^{2}+i z / 2 k\right)} e^{i k(z-c t)}
$$

where $k=\omega / c$ ( $c$ considered here as the light velocity) and $(\rho, \phi, z)$ are the cylindrical coordinates.

The Gaussian beam in Eq.(1) is an ordinary beam, susceptible to a transverse spatial broadening (diffraction). It doubles its initial intensity spot radius, $\Delta \rho_{0}=2 q$, after a distance given by $z_{\text {diff }}=\sqrt{3} k \Delta \rho_{0}^{2}[1]$.

Now, when we make a continuous superposition of equal frequency plane waves with the same amplitude/phase and whose wave vectors lie on a conical surface with half opening angle $\theta$ (that we will call "cone angle"), the resulting wave is the well known ideal non-diffracting zero order Bessel beam $[1,2,5,10]$ :

\footnotetext{
${ }^{\dagger}$ In this case, we also could think that $\Psi$ represents the transverse cartesian component of the electric field.
} 


$$
\Psi(\rho, z, t)=J_{0}(k \sin \theta \rho) e^{i k \cos \theta z} e^{-i \omega t}
$$

which is an exact solution to the wave equation. The central spot of the Bessel beam possesses a radius $\Delta \rho \approx 2.4 /(k \sin \theta)$, and it is preserved during the propagation. Actually, the entire beam transverse structure is preserved indefinitely.

As it was already said, ideal non-diffracting beams carry infinite power. This problem can be overcome, for instance, by considering spatially truncated versions of these beams, i.e., we consider ideal non-diffracting beam solutions truncated, on the plane $z=0$, by a circular aperture of radius $R$.

In this case, the field emanated from the aperture is given (in the paraxial approximation) by the Fresnel diffraction integral[4,11] which, in the case of azimuthal symmetry, assumes the form:

$$
\Psi(\rho, z)=\frac{-i k}{z} e^{i k\left(z+\frac{\rho^{2}}{2 z}\right)} \int_{0}^{R} \Psi_{a p t}\left(\rho^{\prime \prime}\right) e^{\frac{i k \rho^{\prime \prime 2}}{2 z}} J_{0}\left(\frac{k \rho \rho^{\prime \prime}}{z}\right) \rho^{\prime \prime} d \rho^{\prime \prime}
$$

where the time harmonic dependence $e^{-i \omega t}$ is implied. In (33),$\rho^{\prime \prime}$ is the radial coordinate of the aperture and $\Psi_{\text {apt }}\left(\rho^{\prime \prime}\right)$ is the beam on the aperture of radius $R$ localized on the initial plane $z=0$.

When the radius $R$ of the circular aperture is considerably larger than the spot size of the ideal non-diffracting beam which is being truncated, the beam emanated from the aperture (i.e, the truncated beam) will be able to resist the diffraction effects for long (finite) distances.

Figure 1 shows a comparison between a Gaussian and a truncated Bessel beam, both with the same initial spot size. The caption is self explanatory.

Here we define "diffraction resistant beams" as finite power beams that can maintain their spot size for long distances when compared to usual beams. The truncated Bessel beam is an example, but there are many other beams with this characteristic[12-16].

In the following section we present a new way for generating diffraction resistant beams by using a parabolic reflector and a source of spherical waves positioned at a point slightly displaced from its focus (away from the reflector). 


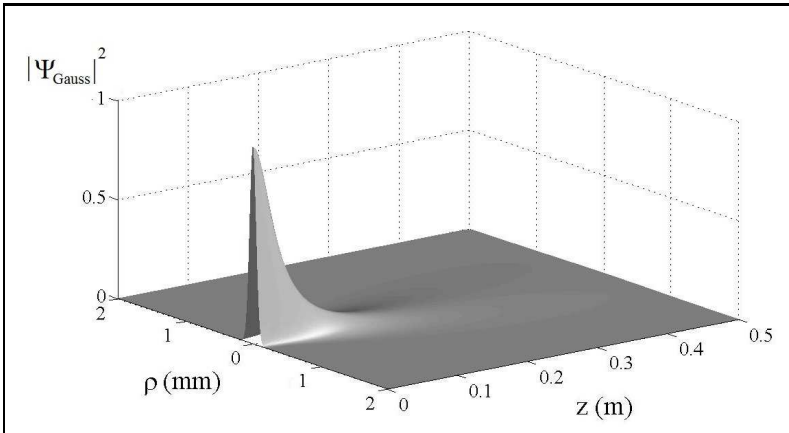

(a)

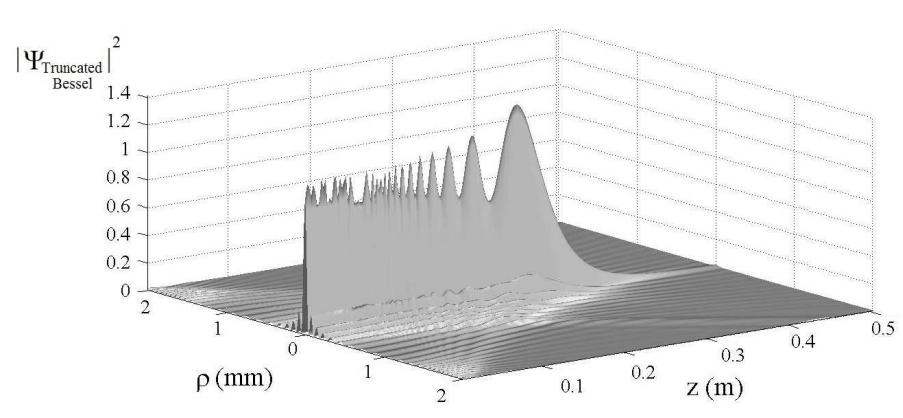

(b)

Figure 1: Comparison of (a) a Gaussian and (b) a Bessel beam truncated by an aperture of $2 \mathrm{~mm}$. Both beams possess $\lambda=850 \mathrm{~nm}$ and spot radius of $68 \mu \mathrm{m}$. The Gaussian beam intensity doubles its initial transverse width after $6 \mathrm{~cm}$, and after $10 \mathrm{~cm}$ its intensity decays by a factor of 10. By contrast, the truncated Bessel beam keeps its transverse shape approximately up to a distance of $33 \mathrm{~cm}$.

\section{Generation of diffraction resistant beams by using a parabolic reflector}

It is well known that in the case of a parabolic reflector, every ray originating from its focus will be reflected in a direction parallel to its axis. Now, if the rays originate from a point slightly displaced away from the reflector, they will converge (after reflection) to its axis, more specifically, they will converge along a focal line[5,7].

As a focal line is a common characteristic of diffraction resistant beams, we are tempted to ask if a parabolic reflector of dimensions much greater than the wavelength considered and with a source of spherical waves positioned at a point slightly displaced away from its focus is capable to generate a diffraction resistant beam within the extended focus range.

We shall analyze the paraboloid setup in three steps. In the first one we use simple ray optics to quantify the range of the focal line. In the second step, we proceed with a heuristic and approximate description of the beam's transverse structure. In spite of the theoretical development at this stage being very approximative, it yields important information about the spot size evolution of the resulting beams, making more understandable the circumstances in which they would present diffraction resistance characteristics.

In the third step, subsection 3.3, we use the Fresnel diffraction integral to describe the 
beams emanated from the paraboloid setup. Examples in optical and microwave frequencies are considered and comparisons with Gaussian beams with the same spot sizes show that these new beams really present resistance to the diffraction effects for long distances.

\subsection{Geometric considerations}

Let us consider a parabolic reflector described in cylindrical coordinates by the equation

$$
z=a \rho^{2}
$$

with $0 \leq z \leq z_{p}$, being $z_{p} \gtrsim z_{f}$ where $z_{f}=1 / 4 a$ is the paraboloid focus position.

In the setup proposed here, the source of spherical waves is positioned at $z=z_{p}$ and the following assumptions are made:

- the dimensions of the parabolic reflector are much greater than the wavelength considered

- the position $z=z_{p} \gtrsim z_{f}$ of the source of spherical waves is such that

$$
0<\frac{z_{p}-z_{f}}{z_{f}}<<1
$$

Figure 2 shows the schematics of the paraboloid setup.

Now, we can use geometric optics to evaluate the line-focus length created by the rays associated with a spherical wave positioned at $z=z_{p}$. The calculations are based on Fig 2 and do not present difficulties[5,7]. The focal line occurs within $D_{\min } \leq z \leq D_{\max }$, where

$$
\begin{gathered}
D_{\text {min }}=\frac{z_{p} z_{f}}{\left(z_{p}-z_{f}\right)} \\
D_{\text {max }}=\frac{z_{p}^{2}+3 z_{p} z_{f}}{z_{p}-z_{f}}=z_{p}+4 D_{\text {min }}
\end{gathered}
$$

Therefore, the line-focus length $Z_{l f l}$ can be written as: 


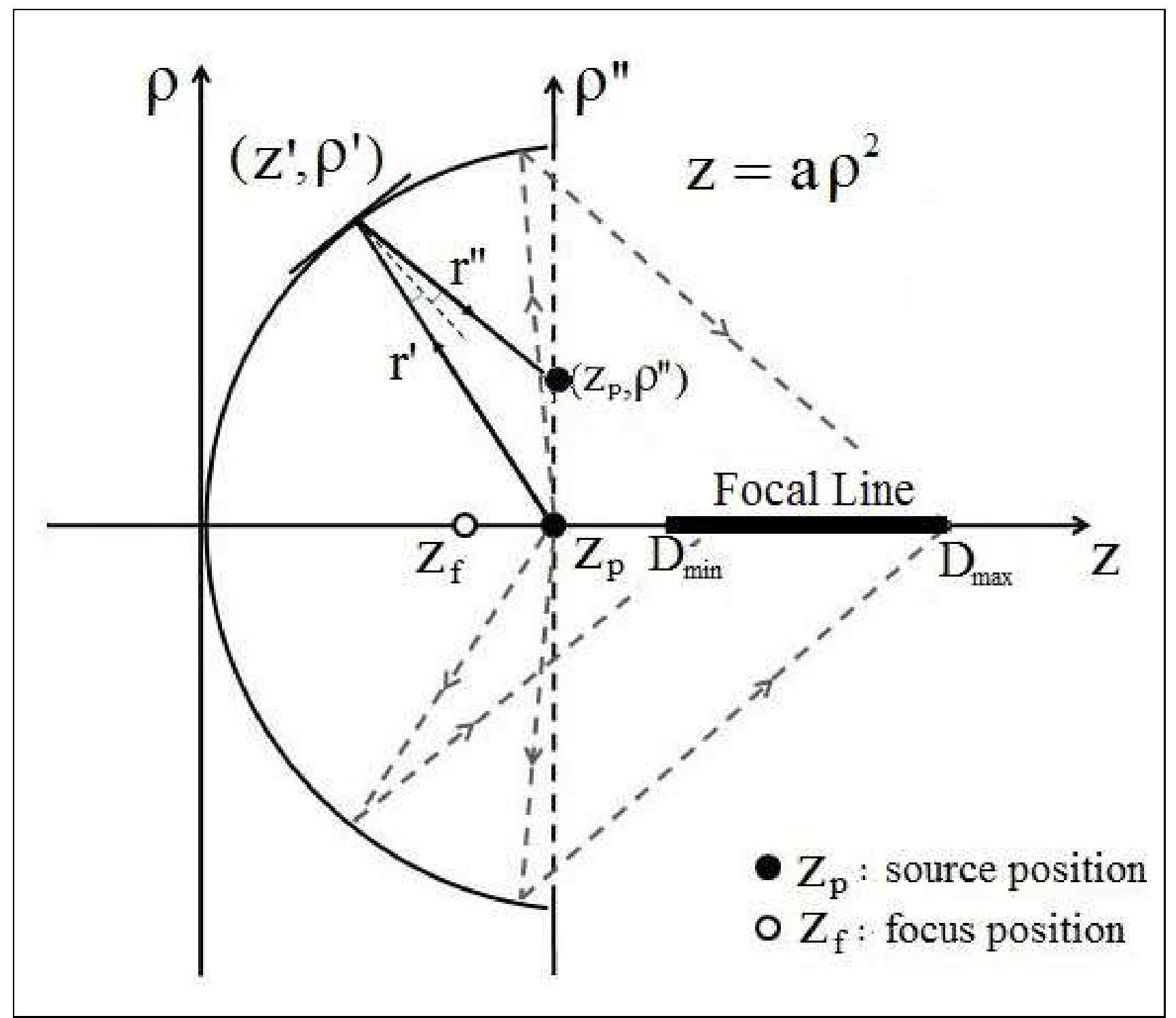

Figure 2: Paraboloid setup: a parabolic reflector with focus in $z=z_{f}$ and a source of spherical waves positioned at $z=z_{p}$, with $z_{p} \gtrsim z_{f}$. 


$$
Z_{l f l}=D_{\max }-D_{\min }=z_{p}+3 D_{\min } \approx 3 D_{\min }
$$

We expect that within the range of the extended focus the resulting beam presents resistance to the diffraction effects. Using plausible assumptions based on geometrical optics and on a spectral characteristic of the zero order Bessel beam, a heuristic derivation about the transverse field pattern of the beams in question will be presented in the next section. From that, we can get important information about the spot size, diffraction resistance length (field depth), etc..

\subsection{Heuristic description of the transverse field pattern of the beams generated by the paraboloid setup}

As we have already said, in this work we have adopted a simplistic analysis, assuming that the field in question, $\Psi(\rho, \phi, z, t)$, is a scalar obeying the usual wave equation.

Let us consider, as already said, a parabolic reflector of dimensions much greater than the wavelength used in the source of the setup. We can imagine a discretization of the paraboloid in belts, which, in turn, are discretized by elements of planes with dimensions much greater than the wavelength of the source of spherical waves [5,7]. See Fig. 3 .

Thus, we can say that the portions of the spherical wave originated from $\left(\rho=0, z=z_{p}\right)$ the "spherical wave center" - that arrive at each one of these planes will be reflected as plane waves with the dimensions of the respective incident planes. So, each point of the $z$ axis, in the extended focus range, will be achieved by a set of "portions" of plane waves from the respective reflector belt

By symmetry, the wave vectors of the set of plane wave "portions" arriving at a point on the focal line will be on the surface of a cone whose half opening angle is given by $\theta$ (see Fig 3 ). In a very approximate way, we can say that this superposition will produce a Bessel beam with cone angle $\theta$, which will propagate by a small interval in $z$ and then "replaced" by the Bessel beam of the next belt, and so on, until the position $D_{\max }$, which marks the end of the focal line.

\footnotetext{
${ }^{\ddagger}$ Each belt of radius $\rho^{\prime}$ on the reflector corresponds to a $z$ point over the axis, according to the reflected rays, as shown in Fig 3 .
} 


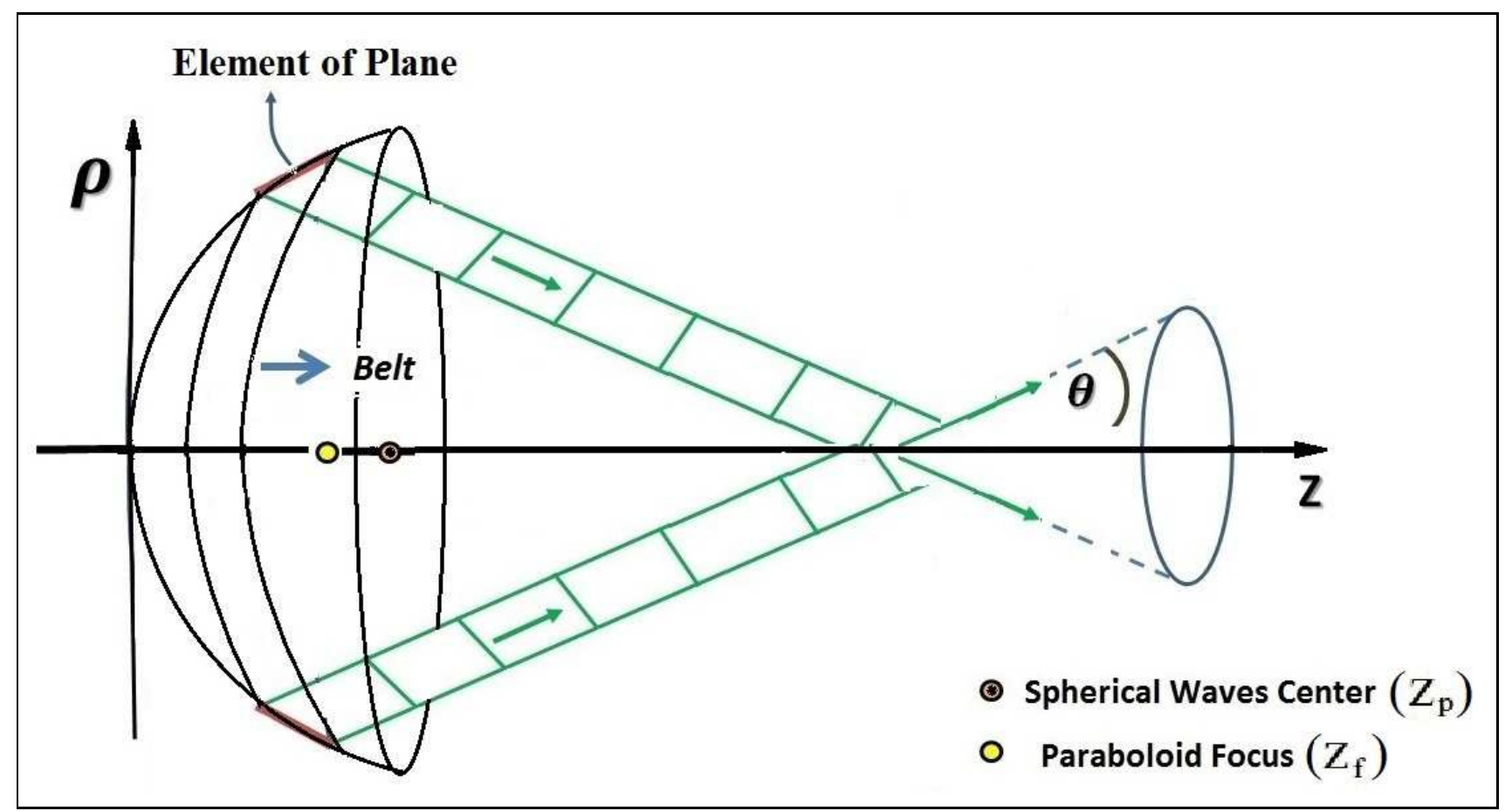

Figure 3: Discretization of the parabolic reflector in belts and the discretization of these belts in elements of plane whose dimensions are taken as being much greater than the wavelength of the source. Each "portion" of the spherical wave that reaches one of these elements of plane is reflected as a "portion" of plane wave that, we assume, does not suffer considerable diffraction until it crosses the $z$-axis. This process results, on each $z$-axis point within the extended focus range, in a superposition of "portions" of plane waves whose wavevectors lie over a conical surface. 
By using geometric optics, it can be shown that

$$
\sin \theta=\frac{1}{\sqrt{\left(\frac{z-\frac{\eta^{2}}{4 z_{f}}}{\eta}\right)^{2}+1}}
$$

where

$$
\eta=2 z_{f} \sqrt{-1+\sqrt{1-\frac{z_{p}}{z_{f}}+\frac{z_{p}-z_{f}}{z_{f}^{2}} z}}
$$

and $D_{\min }<z \leq D_{\max }$.

According to these general considerations and noting that the intuitive scheme above does not provide any information about the beam amplitude on different z positions, we conjecture that within the focal line and for points near the z-axis, the resulting beam can be approximately written as $\Psi(\rho, z, t) \approx A(z) J_{0}(k \sin \theta \rho) \exp (i k \cos \theta z) \exp (-i \omega t)$, where $A(z)$ is an (unknown) amplitude function depending on $z$. In this way, we can get a very useful information about the transverse behavior of the resulting beam, for $D_{\min }<z \leq D_{\max }$ and for points near the z-axis, by considering the ratio $\Psi(\rho, z, t) / \Psi(\rho=0, z, t)$ which, in this approach, is given by

$$
\frac{\Psi(\rho, z, t)}{\Psi(\rho=0, z, t)} \approx J_{0}(k \sin \theta \rho)
$$

where it should be noted that $\theta$ is not constant but dependent on $z$, according to Eqs.(9]10).

Equation (11) can furnish important information about the diffraction resistance characteristics of the beams generated from this setup. Specifically, the spot size radius $-\Delta \rho-$ of the resulting beam can be estimated as

$$
\Delta \rho \approx \frac{2.4}{k \sin \theta}
$$

which is clearly dependent of $z$ due to Eqs.(910).

The expression for the spot radius is complicated, but many simplifications can be done in the cases where the relation (5) is satisfied and, as we have already said, these are the cases of interest here. The following results of this subsection are obtained assuming relation (5). 
It is possible to show that the minimum value of $\Delta \rho$, the beam waist, is approximately given by

$$
\Delta \rho_{\min } \approx 0.6 \frac{z_{p}}{z_{p}-z_{f}} \lambda
$$

and occurs at $z=z_{b w}$, with

$$
z_{b w} \approx \frac{16}{9} \frac{z_{f}^{2}}{z_{p}-z_{f}} \approx 1.77 D_{\text {min }}
$$

Within the extended focus range, the beam spot radius, after $z=z_{b w}$, increases until reaching its maximum value at $z=D_{\max }$, with $D_{\max }$ given by Eq.(7). By using this value of $z$ into Eq.(12) we get

$$
\Delta \rho_{\max } \approx 0.38 \frac{z_{p}+z_{f}}{z_{p}-z_{f}} \lambda
$$

So, after the distance

$$
Z=D_{\max }-z_{b w} \approx 2.22 D_{\min }=2.22 \frac{z_{p} z_{f}}{z_{p}-z_{f}}=0.6 z_{f} \Delta \rho_{\min } k
$$

we have that

$$
\frac{\Delta \rho_{\max }}{\Delta \rho_{\min }} \approx 1.26
$$

We consider $\$ Z$, Eq.(16), as the field depth of the resulting beam. After this distance, its spot radius becomes 1.26 times greater than the beam waist, which occurs at $z=z_{b w}$.

This field depth can be considerably greater than the diffraction distance (Rayleigh distance) of the Gaussian beam, given by $Z_{\text {Gauss }}=\sqrt{3} \Delta \rho_{\text {Gauss }}^{2} k$, where $\Delta \rho_{\text {Gauss }}$ is the Gaussian beam's waist. To see this, let us consider a situation where the waists of a Gaussian beam and of a beam generated by the paraboloid setup are equal, i.e., $\Delta \rho_{\text {Gauss }}=\Delta \rho_{\text {min }}$. In this case we have:

$\S$ It is important to note that in Eq.(16) for the field depth, the beam waist $\Delta \rho_{\min }$ also depends on $z_{f}-$ see Eq. (13)

IThe distance wherein the Gaussian beam intensity doubles the value of its waist. 


$$
\frac{Z}{Z_{\text {Gauss }}} \approx 0.35 \frac{z_{f}}{\Delta \rho_{\min }}
$$

and it is not difficult to see that the above ratio may reach values significantly greater than unity.

For instance, let us assume a parabolic reflector whose focus is localized at $z_{f}=2 \mathrm{~cm}$ and a spherical wave source, with $\lambda=850 \mathrm{~nm}$, located at a distance of $8 \lambda$ away from the focus, i.e., $z_{p}=z_{f}+8 \lambda$. In this case, using Eq.(6) and Eq.(17), we obtain the extended focus occurring between $D_{\min }=58.8 \mathrm{~m}$ and $D_{\max }=235.4 \mathrm{~m}$, respectively. Yet, according to our approximative equations, the resulting beam waist will be $\Delta \rho_{\min } \approx 1.5 \mathrm{~mm}$, localized at $z_{b w} \approx 104 \mathrm{~m}$ and, from there, after a distance of $Z=131 \mathrm{~m}$ (the field depth), the spot radius will be $\Delta \rho_{\max } \approx 1.9 \mathrm{~mm}$, just 1.26 times greater. A Gaussian beam with the same wavelength would double this same waist after a distance $Z_{\text {Gauss }} \approx 29 \mathrm{~m}$.

Figures 4 and 5 show, respectively, the evolutions of the beam's transverse pattern, according Eq.(11) and of the beam's spot radius, according Eq.(12), both complemented by eqs.(9010).

From these figures, we can see that the beam has a diffraction resistance behavior for a distance even greater than $Z=131 \mathrm{~m}$, actually for a distance approximately equal to the line-focus length $Z_{l f l}=176 \mathrm{~m}$.

While in this section we have presented a heuristic description of the beam's transverse evolution based on Eq.(11), in the following section the full resulting beam emanated from the paraboloid setup is obtained in a more rigorous way by using the wave theory. More specifically, we first evaluate the field on the paraboloid aperture and from it the emanated field is calculated through the Fresnel diffraction integral. Some examples are presented.

\subsection{Description of the beams resulting from the paraboloid setup by using wave optics}

Let us consider the parabolic setup represented by Fig,2, where a spherical wave source is located at $\left(\rho=0, z=z_{p}\right)$, with $z_{p} \gtrsim z_{f}=1 / 4 a$. The wave produced by this source is written as: 


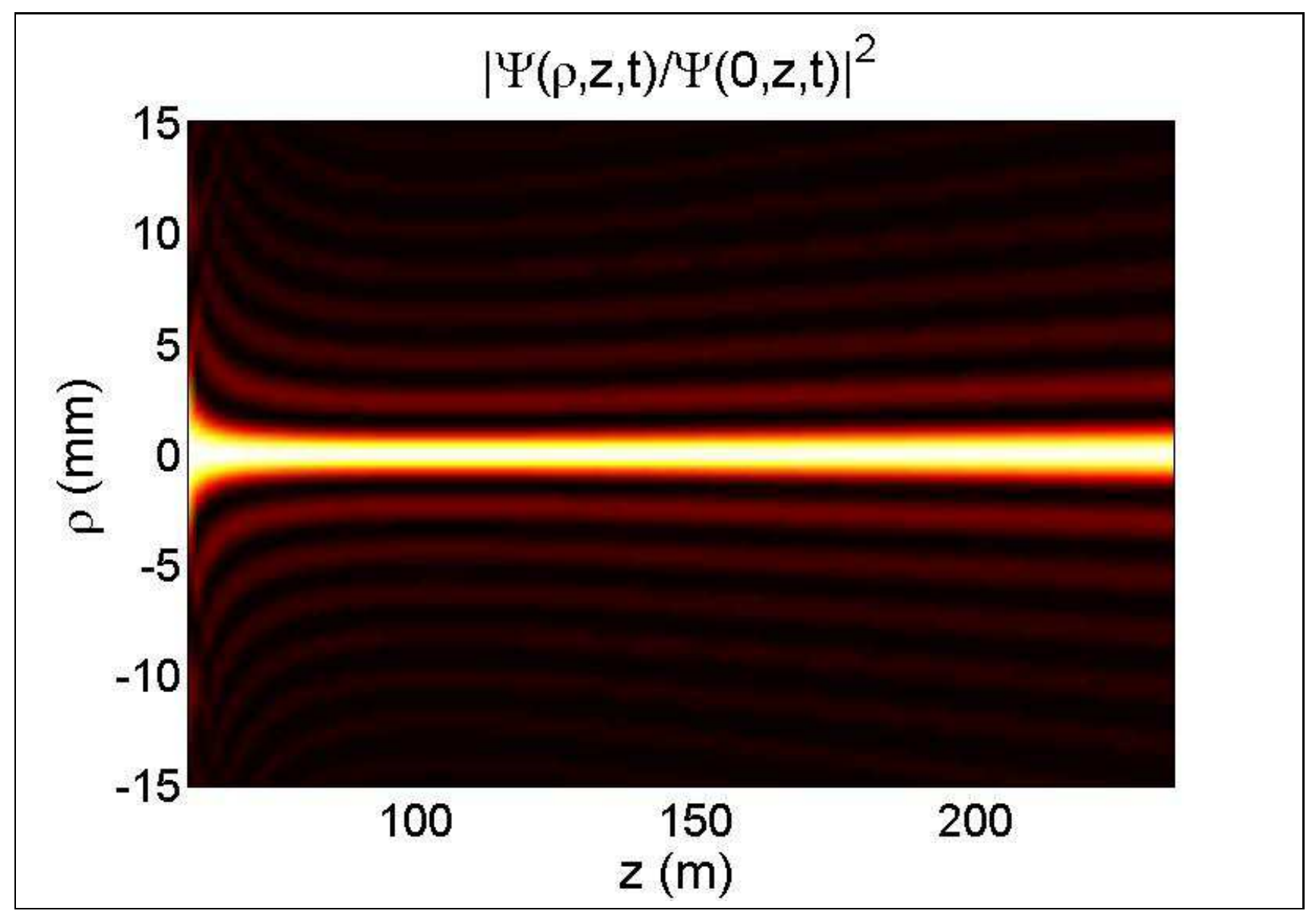

Figure 4: Evolution of the beam transverse pattern, $\Psi(\rho, z, t) / \Psi(\rho=0, z, t)$, according Eq.(11), with eqs.(9 10), considering a source of spherical waves with $\lambda=850 \mathrm{~nm}$, displaced $8 \lambda$ away from a parabolic reflector whose focus is located at $z_{f}=2 \mathrm{~cm}$. 


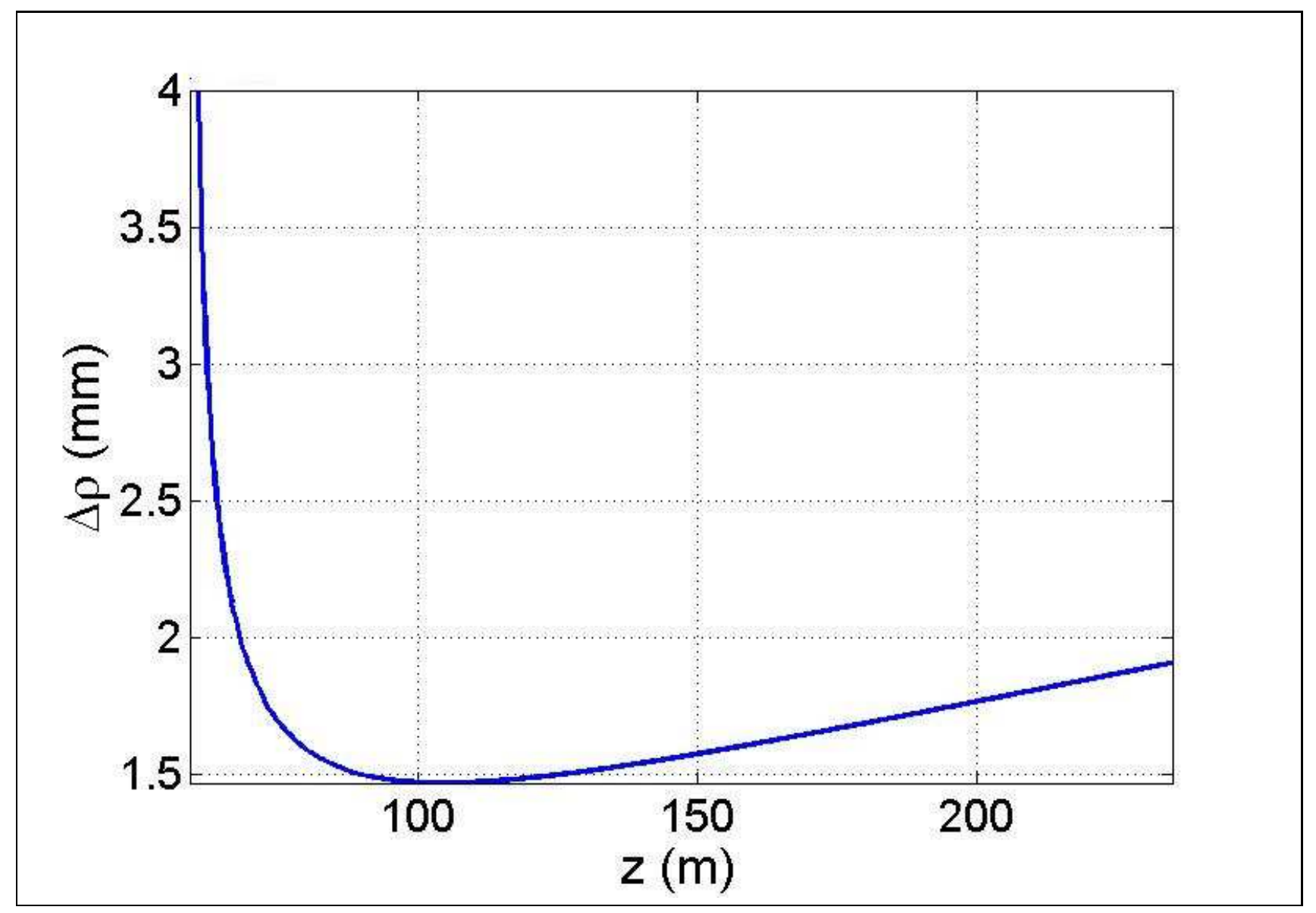

Figure 5: Variation of the spot radius within the extended focus range, according to Eq.(12), considering a source of spherical waves with $\lambda=850 \mathrm{~nm}$, displaced $8 \lambda$ away from a parabolic reflector focus which is located at $z_{f}=2 \mathrm{~cm}$. 


$$
\Psi_{s p h}=\frac{e^{i k r}}{r}
$$

where $r$ is the distance from the source to the point in question.

So, the incident wave on the paraboloid reflector, represented by the coordinates $\left(\rho^{\prime}, \phi^{\prime}, z^{\prime}\right)$ with $z^{\prime}=a \rho^{\prime 2}$, is given by

$$
\Psi_{i}=\frac{e^{i k r^{\prime}}}{r^{\prime}}
$$

where

$$
r^{\prime}=\sqrt{\left(z_{p}-z^{\prime}\right)^{2}+\rho^{\prime 2}}
$$

We will use this incident wave to evaluate, approximately, the field on the paraboloid aperture, that is, on the circular aperture of radius $R=\sqrt{z_{p} / a}$. To achieve this, first we note that a ray originating at the source position and reflected at the paraboloid point $\left(\rho^{\prime}, \phi^{\prime}, z^{\prime}\right)$, will travel a distance $r^{\prime \prime}$, with

$$
r^{\prime \prime}=\sqrt{\left(z_{p}-z^{\prime}\right)^{2}+\left(\rho^{\prime}-\rho^{\prime \prime}\right)^{2}},
$$

and reach the plane $z=z_{p}$ (where the paraboloid aperture is) at the point $\left(\rho^{\prime \prime}, \phi^{\prime \prime}\right)$, where $\phi^{\prime \prime}=\phi^{\prime}$ and

$$
\rho^{\prime \prime}=\frac{4 z_{f}\left(z_{p}-z_{f}\right)\left(4 z_{p} z_{f}-\rho^{\prime 2}\right) \rho^{\prime}}{\left(\rho^{\prime 2}-4 z_{p} z_{f}\right)\left(\rho^{\prime 2}-4 z_{f}^{2}\right)+16 \rho^{\prime 2} z_{f}^{2}}+\rho^{\prime} .
$$

Based on the assumption that the paraboloid dimensions are much greater than the wavelength, the next step is to consider that each little portion of the incident spherical wave $\Psi_{i}$, Eq.(20), will be reflected as a plane wave, in such way that the reflected field on the paraboloid aperture, $\Psi_{\text {apt }}$, can be approximately written as

$$
\Psi_{a p t}=-\Psi_{i} e^{i k r^{\prime \prime}}=-\frac{e^{i k\left(r^{\prime}+r^{\prime \prime}\right)}}{r^{\prime}}
$$

where $r^{\prime}$ and $r^{\prime \prime}$ are given by eqs.(21,22). 
Now, with the knowledge of the reflected field on the paraboloid aperture, we can evaluate the emanated field by using the Fresnel diffraction integral given by Eq.(3).

It is important to notice that this diffraction integral is performed over the aperture's radial coordinate, $\rho^{\prime \prime}$, and so the $\Psi_{a p t}$, Eq.(24), should be written in terms of this variable by inverting the relation (23)). Such inversion is a difficult task, therefore we choose to change the variable of integration in Eq.(3) from $\rho^{\prime \prime}$ to $\rho^{\prime}$, by using directly Eq.(23).

With this, the resulting beam emanated from the paraboloid setup can be obtained by numerically solving the Fresnel diffraction integral over the variable $\rho^{\prime}$ :

$$
\begin{aligned}
\Psi(\rho, z)= & \frac{i k}{z-z_{p}} e^{i k\left[\left(z-z_{p}\right)+\frac{\rho^{2}}{2\left(z-z_{p}\right)}\right]} \\
& \times \int_{0}^{R} \frac{e^{i k\left[r^{\prime}\left(\rho^{\prime}\right)+r^{\prime \prime}\left(\rho^{\prime}\right)\right]}}{r^{\prime}\left(\rho^{\prime}\right)} e^{\frac{i k\left[\rho^{\prime \prime}\left(\rho^{\prime}\right)\right]^{2}}{2\left(z-z_{p}\right)}} J_{0}\left(\frac{k \rho \rho^{\prime \prime}\left(\rho^{\prime}\right)}{z-z_{p}}\right) \rho^{\prime \prime}\left(\rho^{\prime}\right) \frac{d \rho^{\prime \prime}}{d \rho^{\prime}} d \rho^{\prime},
\end{aligned}
$$

where the functions of $\rho^{\prime}: \rho^{\prime \prime}\left(\rho^{\prime}\right)$ and $d \rho^{\prime \prime} / d \rho^{\prime}$ are obtained from Eq.(23), $r^{\prime}\left(\rho^{\prime}\right)$ and $r^{\prime \prime}\left(\rho^{\prime}\right)$ are given by eqs.(21) and (22), the last one complemented by Eq.(23).

Now that we have the integral solution for the resulting beam, we are going to applied it to a few examples, which will confirm the beam's diffraction resistance properties as well the predictions of the previous sections/subsections.

\section{First example:}

Let us use Eq.(25) for describing the generation of a beam with $\lambda=850 \mathrm{~nm}$ by the paraboloid setup whose focus $z_{f}=2 \mathrm{~cm}$. The source of spherical waves is located $8 \lambda$ away from the focus, i.e., $z_{p}=z_{f}+8 \lambda$, which implies an aperture of radius $R=4 \mathrm{~cm}$.

With these parameters, our approximative equations (6],7],8]12]13]14]15) predict: i) a linefocus length $Z_{l f l}=175.5 \mathrm{~m}$, inside the interval $D_{\min }=58.8 \mathrm{~m} \leq \mathrm{z} \leq \mathrm{D}_{\max }=235.4 \mathrm{~m}$; ii) a beam waist of $\Delta \rho_{\min } \approx 1.5 \mathrm{~mm}$ at $z_{b w} \approx 104 \mathrm{~m}$, with a diffraction resistance behavior for (at least) a distance of $Z \approx 131 \mathrm{~m}$.

The resulting beam is evaluated by a numerical simulation of the diffraction integral, Eq.(25) and the results are shown in Figures 6 and 7.

Figure 6a shows the beam intensity and Fig.6b its orthogonal projection. We can see that 
the extended focus occurs as predicted, but due to the intensity variation along the distance, the details about the spot size behavior are not very clear, although it is clear its resistance to the diffraction effects along the extended focus range.

Now, to get more information about the transverse beam behavior, Fig.7a shows the intensity ratio $|\Psi(\rho, z, t) / \Psi(\rho=0, z, t)|^{2}$ for the resulting beam starting from $z=D_{\text {min }}=58.8 \mathrm{~m}$. The evolution of the spot size within the extended focus range $D_{\min }<z \leq D_{\max }$ is satisfactorily described by Eq.(12) with eqs.(9,10), as we can see by comparing Figures 6 and 7a. We can clearly see that the size and position of the beam waist agree with the predicted values and it is also very clear that the beam is very resistant to the diffraction effects until the distance $Z \approx 131 \mathrm{~m}$. Actually its central spot suffers just small variations for distances greater than the predicted field depth $Z$, but it is also clear that after that the beam's lateral lobes become much more pronounced $\|$.

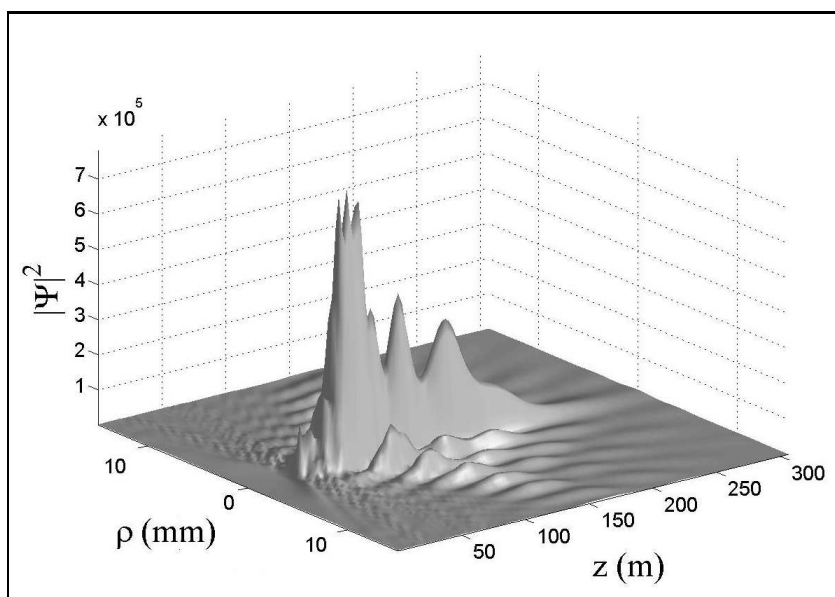

(a)

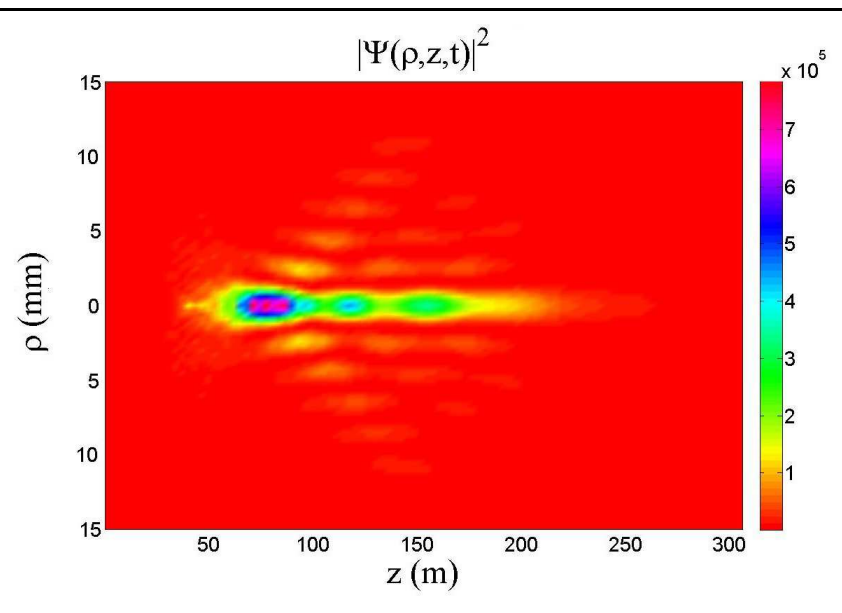

(b)

Figure 6: (a)Evolution of the beam intensity resulting from the paraboloid setup when $\lambda=$ 850nm, $z_{f}=2 \mathrm{~cm}$ and $z_{p}=z_{f}+8 \lambda$; (b) The orthogonal projection of the resulting beam intensity. We can see, as predicted, that the beam possesses a extended focus range in which it presents resistance to the diffraction effects.

Figure $7 \mathrm{~b}$ shows the same kind of intensity ratio for a Gaussian beam with the same waist at the same position. After $29 \mathrm{~m}$ this beam doubles its intensity spot size.

\footnotetext{
"It is very interesting that the beam central spot continues to suffer just small variations after the predicted field depth, even if the side lobes become more pronounced.
} 


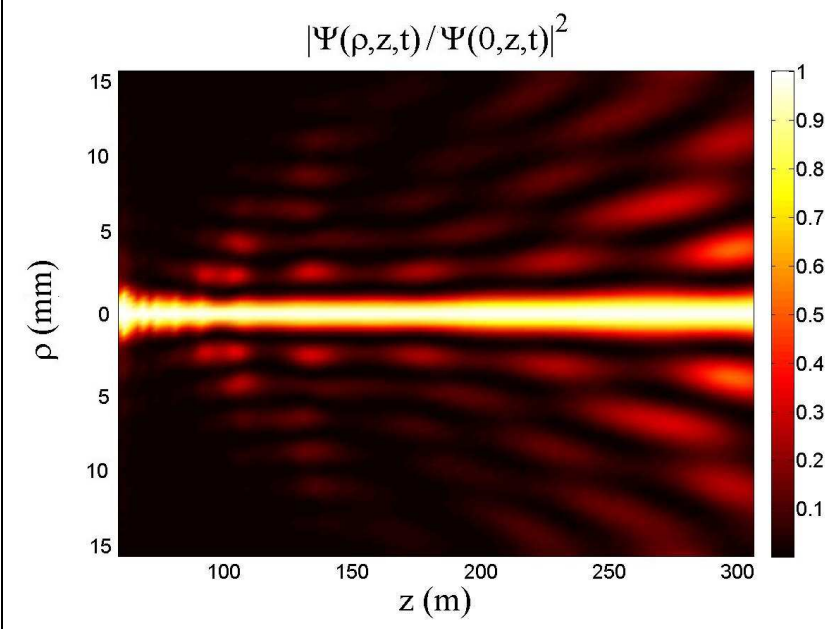

(a)

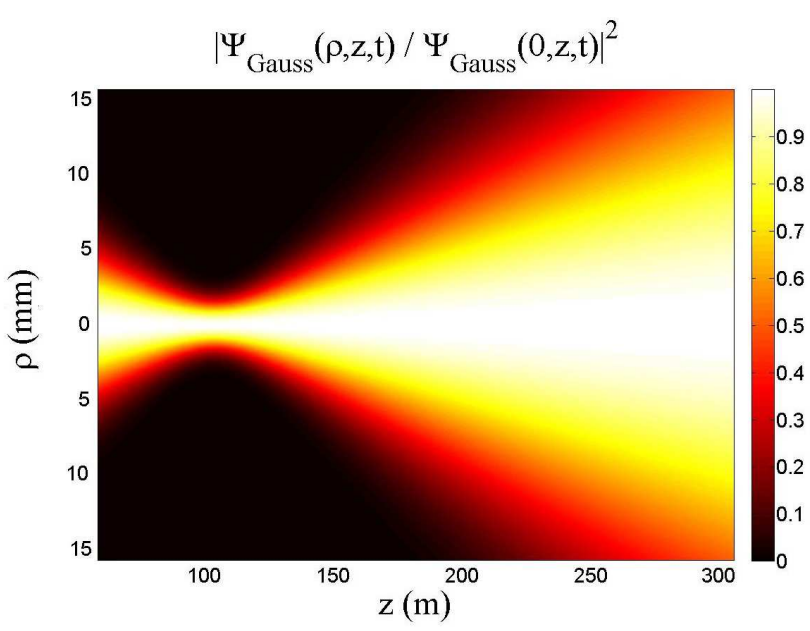

(b)

Figure 7: (a)The intensity ratio $|\Psi(\rho, z, t) / \Psi(\rho=0, z, t)|^{2}$ for the resulting beam starting from $z=D_{\min }=58.8 \mathrm{~m}$. We can see that the size and position of the beam waist agree with the predicted values and it is also very clear that the beam is very resistant to the diffraction effects for distances even greater than $Z \approx 131 \mathrm{~m}$; (b) The same kind of intensity ratio for a Gaussian beam with the same waist at the same position. After $29 \mathrm{~m}$ this beam doubles its intensity spot size.

\section{Second example:}

Now, let us consider our paraboloid setup to generate a beam of wavelength $\lambda=1 \mathrm{~cm}$. In this case we choose the focus $z_{f}=0.5 \mathrm{~m}$. The source of spherical waves is located at $z_{p}=0.525 \mathrm{~m}$, which implies an aperture of radius $R \approx 1 \mathrm{~m}$.

For this configuration our approximative equations (6,7,8,12,13,14,15) predict: i) a linefocus length $Z_{l f l}=32 \mathrm{~m}$, inside the interval $D_{\min }=10.5 \mathrm{~m} \leq \mathrm{z} \leq \mathrm{D}_{\max }=42.5 \mathrm{~m}$; ii) a beam waist of $\Delta \rho_{\min } \approx 12.6 \mathrm{~cm}$ at $z_{b w} \approx 18.6 \mathrm{~m}$, with a diffraction resistance behavior for (at least) a distance of $Z \approx 24 \mathrm{~m}$.

We evaluated the resulting beam by the numerical simulation of the diffraction integral, Eq.(25), and the results are shown in Figures 8 and 9.

Figure 8 shows the beam intensity and we can see that the extended focus occurs as predicted.

As before, to get more information about the transverse beam behavior, Fig.9a shows the intensity ratio $|\Psi(\rho, z, t) / \Psi(\rho=0, z, t)|^{2}$ for the resulting beam starting from $z=D_{\min }=$ 


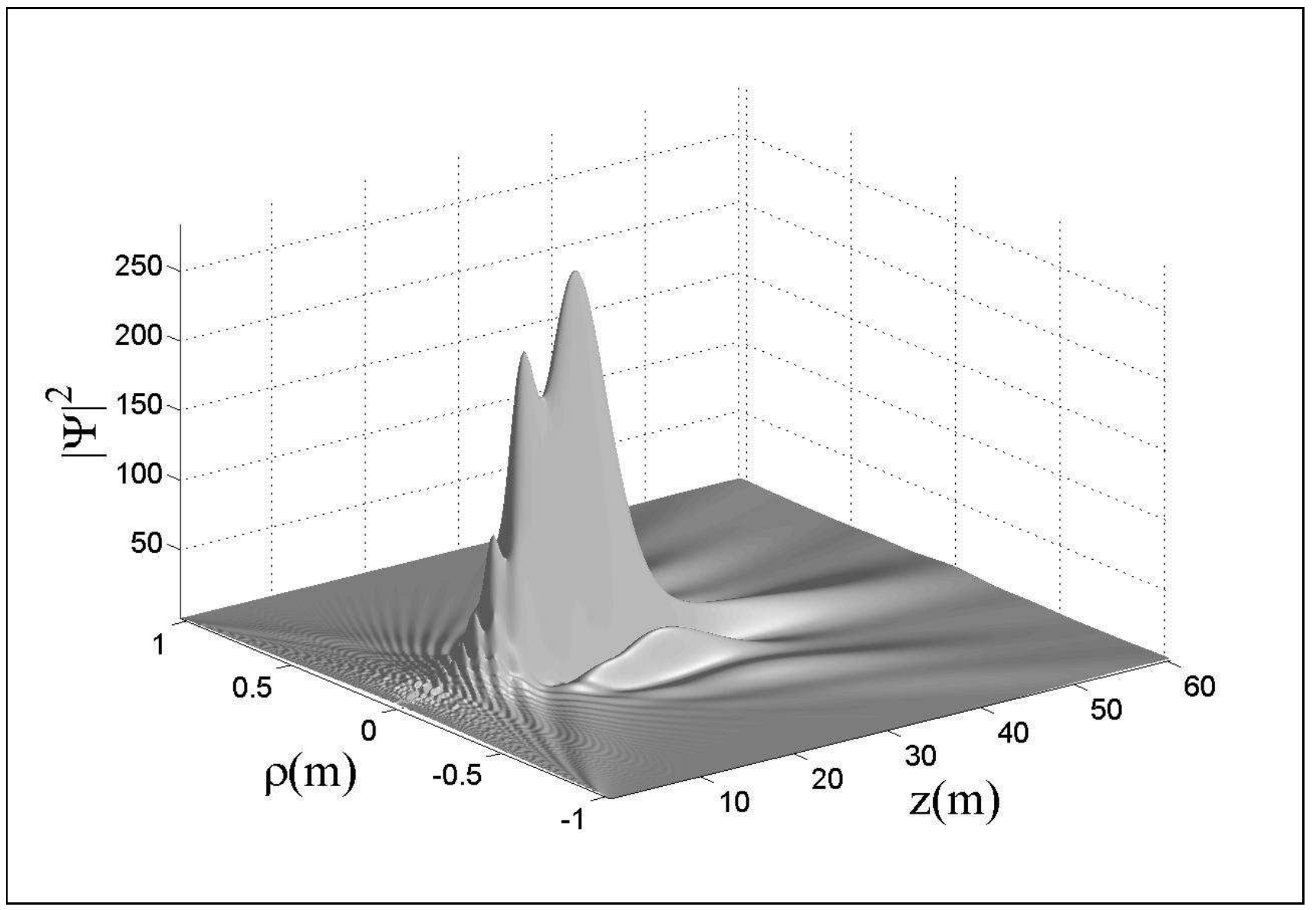

Figure 8: (a) Evolution of the beam intensity resulting from the paraboloid setup when $\lambda=1 \mathrm{~cm}$, $z_{f}=0.5 \mathrm{~m}$ and $z_{p}=0.525 \mathrm{~m}$. 


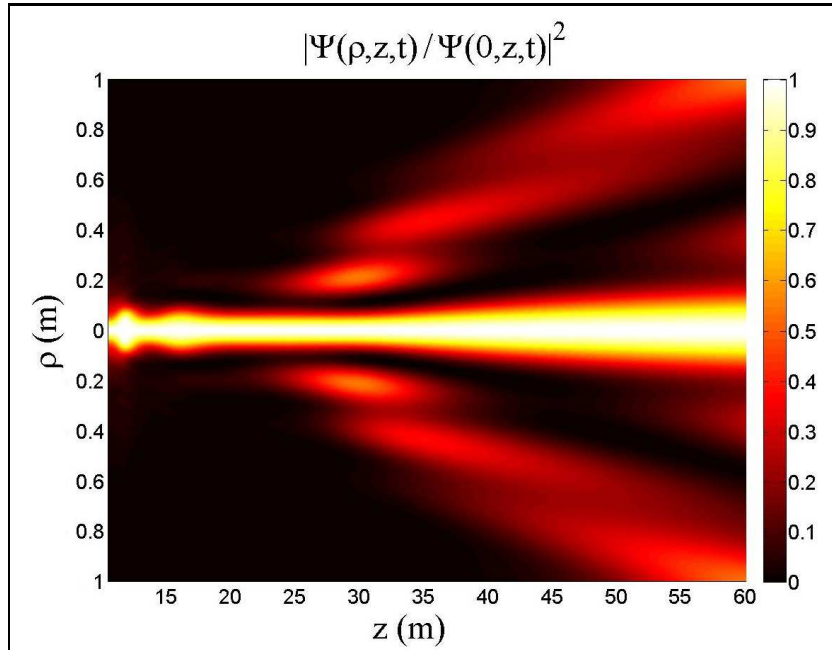

(a)

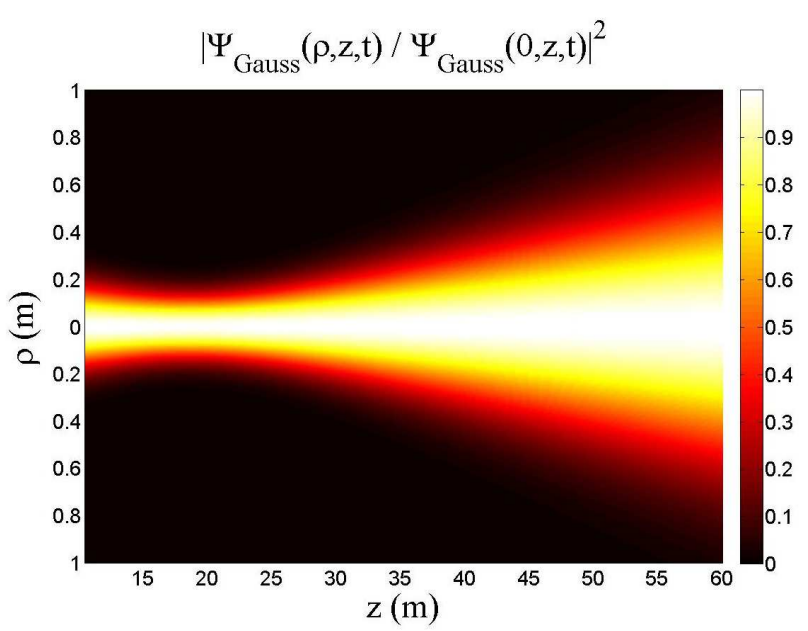

(b)

Figure 9: (a) Intensity ratio $|\Psi(\rho, z, t) / \Psi(\rho=0, z, t)|^{2}$ for the resulting beam starting from $z=D_{\min }=10.5 \mathrm{~m}$. The beam demonstrates resistance to the diffraction effects for distances even greater than $Z \approx 42.5 \mathrm{~m}$; (b) The same kind of intensity ratio for a Gaussian beam with the same waist at the same position. After a distance of $17 \mathrm{~m}$ this beam doubles its intensity spot size

$10.5 \mathrm{~m}$. Except for a small initial oscillation of the spot radius, the evolution of the spot size within the extended focus range is satisfactorily described by Eq.(12) with eqs.(9]10), being the size and position of the beam waist in agreement with the predicted values. The beam demonstrates resistance to the diffraction effects until the distance $Z \approx 42.5 \mathrm{~m}$. Actually its central spot suffers just small variations for distances greater than the predicted field depth $Z$.

Figure $9 \mathrm{~b}$ shows the same kind of intensity ratio for a Gaussian beam with the same waist at the same position. After a distance of $17 \mathrm{~m}$ this beam doubles its intensity spot size.

The above examples in the optical and microwave frequency ranges confirm our predictions about the possibilities of generating diffraction resistant beams through using a parabolic reflector and a source of spherical waves slightly displaced from its focus.

\section{Conclusion}

This paper presents a simple way to generate diffraction resistant scalar beams by using as hypothetical experimental setup a parabolic reflector and a source of spherical waves located 
at a position slightly displaced away from the focus of the reflector.

We have obtained a set of approximative equations describing some important characteristics of the new beams, like the extended focus range, the evolution of the beam spot size, the diffraction resistance length, etc.. We also have obtained the respective Fresnel diffraction integral which describes the resulting beams and two examples have been provided, in optical and microwave frequencies, confirming our predictions.

Due to its simplicity and low-cost characteristic, the method here presented can be a very interesting alternative for the generation of long range diffraction resistant beams in optics and microwave applications.

A more complete and rigorous analysis of the setup proposed here, taking into account the vectorial nature of the fields, will be addressed in a future paper.

This work was supported by FAPESP (under grants 2013/26437-6 and 2014/04867-1); CNPq (under grant 312376/2013-8) and CAPES.

The authors thank Erasmo Recami, Ioannis M. Besieris and Massimo Balma for valuable discussions and kind collaboration.

\section{References}

[1] H. E. Hernández-Figueroa, M. Zamboni-Rached and E. Recami, Localized Waves, 1st ed. Hoboken, USA: John Wiley \& Sons, 2008.

[2] J. Durnin, J. J. Miceli and J. H. Eberly, Diffraction-free beams, Phy.Rev.Lett. 58, 14991501, 1987.

[3] I. S. Gradshteyn and I. M. Ryzhik, Table of Integrals, Series and Products, 5th ed. USA: Academic Press, 1996.

[4] J. Goodman, Introduction to Fourier Optics, 2nd ed. USA: McGraw-Hill, 1996.

[5] M. C. Assis, Parabolic reflectors used in generation of non-diffracting beams, M.Sc. thesis. Campinas State University, 2013. 
[6] J. W. M. Baars, The Paraboloidal Reflector Antenna in Radio Astronomy and Communication, 1st ed. USA: Springer, 2007.

[7] Michel Zamboni-Rached and Erasmo Recami "Parabolic antennas, and circular slot arrays, for the generation of Non-Diffracting Beams of Microwaves," arXiv:physics/1408.5635 vl, Aug. 24, 2014.

[8] L. A. Ambrósio, Localized beams in optical tweezers with conventional and metamaterial particles, Ph.D thesis. Campinas State University, 2010.

[9] L. Stanislav, On the generation and application of localized waves, M.Sc. thesis. Virginia Polytechnic Institute, 2001.

[10] M. Zamboni-Rached, Ondas localizadas aplicadas aos meios difrativos/dispersivos, Ph.D thesis. Campinas State University, 2004

[11] F.Gori, G. Guattari and C.Padovani, Bessel-Gauss Beams, Optics Communications. 64, 491-495, 1987.

[12] Michel Zamboni-Rached, Leonardo A. Ambrósio, and Hugo E. Hernández-Figueroa, "Diffraction-attenuation resistant beams: their higher order versions and finite-aperture generations," Appl. Opt. 49, 5861-5869 (2010).

[13] G. A. Siviloglou and D. N. Christodoulides, "Accelerating finite energy Airy beams," Opt. Lett. 32, 979-981 (2007)

[14] Michel Zamboni-Rached, K. Z. Nóbrega, and C. A. Dartora, "Analytic description of Airytype beams when truncated by finite apertures," Opt. Express 20, 19972-19977 (2012).

[15] Ioannis M. Besieris and Amr M. Shaarawi, "Accelerating Airy beams with non-parabolic trajectories," Optics Communications, Vol. 331, 235-238 (2014).

[16] I. M. Besieris and A. M. Shaarawi, "Localized monochromatic and pulsed waves in hyperbolic metamaterials (invited paper)," Progress In Electromagnetics Research, Vol. 143, 761-771, 2013. 